\title{
Tungsten Promoted Dearomatization of Heterocycles: Uncovering the Latent 2-Azadiene Character of Pyrimidines
}

\author{
Yogesh Surendranath, Bradley W. Nash, W. Hill Harman, William H. Myers," Michal \\ Sabat, and W. Dean Harman*
}

Table of Contents:

Experimental Details............................S1

Spectral Data for 2 - 5..........................S4

\section{Experimental Details.}

NMR spectra were obtained on a 300 or $500 \mathrm{MHz}$ Varian INOVA spectrometer. All chemical shifts are reported in ppm and are referenced to tetramethylsilane (TMS) utilizing residual ${ }^{1} \mathrm{H}$ or ${ }^{13} \mathrm{C}$ signals of the deuterated solvents as an internal standard. Coupling constants $(J)$ are reported in hertz $(\mathrm{Hz})$. Resonances in the ${ }^{1} \mathrm{H}$ NMR due to pyrazole ligands (Tp) are listed by chemical shift and multiplicity only (all coupling constants are $2 \mathrm{~Hz}$ unless otherwise noted). Infrared spectra (IR) were recorded on a MIDAC Prospect Series (Model PRS) spectrometer as a glaze on a Horizontal Attenuated Total Reflectance (HATR) accessory (Pike Industries). Electrochemical experiments were performed under a dinitrogen atmosphere using a BAS Epsilon EC-2000 potentiostat. Cyclic voltammetry data was taken at ambient temperature at $100 \mathrm{mV} / \mathrm{s}(25$ ${ }^{\circ} \mathrm{C}$ ) (unless otherwise specified) in a standard three-electrode cell from +1.7 to $-1.7 \mathrm{~V}$ with a glassy carbon working electrode, $N, N$-dimethylacetamide (DMA) solvent, and tetrabutylammonium hexaflurophosphate $(\mathrm{TBAH})$ electrolyte $(\sim 0.5 \mathrm{M})$. All potentials are reported versus NHE (Normal Hydrogen Electrode) using cobaltocenium hexafluorophosphate $\left(\mathrm{E}_{1 / 2}=-0.78 \mathrm{~V}\right)$ or ferrocene $\left(\mathrm{E}_{1 / 2}=0.55 \mathrm{~V}\right)$ as an internal standard. The peak-to-peak separation was less than $100 \mathrm{mV}$ for all reversible couples. Mass spectra were obtained on either a JEOL JMS600 using $\mathrm{FAB}^{+}$, or a Finnagan MAT TSQ7000 using ESI ${ }^{+}$, no counter ions were observed. Elemental analyses (EA) were obtained from Atlantic Microlabs, Inc. Unless otherwise noted, all synthetic reactions and electrochemical experiments were performed under a dry nitrogen atmosphere. $\mathrm{CH}_{2} \mathrm{Cl}_{2}$, benzene, THF (tetrahydrofuran), and hexanes were purged with nitrogen and purified by passage through a column packed with activated alumina. Other solvents and liquid reagents were thoroughly purged with nitrogen and passed through a column packed with activated basic alumina prior to use. Deuterated solvents were used as received from Cambridge Isotopes.

$\mathrm{TpW}(\mathrm{NO})\left(\mathrm{PMe}_{3}\right)(\kappa N$-pyrimidine) (2)

A homogenous solution of pyrimidine $(0.543 \mathrm{~g}, 6.78 \mathrm{mmol})$ in $17.1 \mathrm{~mL}$ DME was added to complex 1 ( $2.020 \mathrm{~g}, 3.48 \mathrm{mmol})$. The resulting solution was allowed to sit for $21 \mathrm{hrs}$. The reaction solution was then added to $250 \mathrm{~mL}$ of stirring pentane and the resulting precipitate was collected on a medium porosity glass filter and dried in vacuo to give $\mathbf{2}$ $\left(1.806 \mathrm{~g}, 3.10 \mathrm{mmol}, 89 \%\right.$ yield) as a blue powder. IR (sealed KBr Plates, glaze): $v_{\mathrm{NO}}=$ $1515 \mathrm{~cm}^{-1}$. CV (DMA, TBAH, $100 \mathrm{mV} / \mathrm{s}$, vs. NHE): $\mathrm{E}_{1 / 2}=-0.66 \mathrm{~V} .{ }^{1} \mathrm{H}$ NMR (acetone- $d_{6}$, ঠ): $7.94(1 \mathrm{H}, \mathrm{d}, \mathrm{Tp}(3,5)), 7.93(1 \mathrm{H}, \mathrm{d}, \mathrm{Tp}(3,5)), 7.89(1 \mathrm{H}, \mathrm{d}, \mathrm{Tp}(3,5)), 7.82(1 \mathrm{H}, \mathrm{d}, \mathrm{Tp}$ 
$(3,5)), 7.53(2 \mathrm{H}, \mathrm{d}, 2 \mathrm{Tp}(3,5)), 6.36(1 \mathrm{H}, \mathrm{t}, \mathrm{Tp}(4)), 6.34(1 \mathrm{H}, \mathrm{t}, \mathrm{Tp}(4)), 6.34 \pm 0.04(1 \mathrm{H}$, $\mathrm{d}(J=3.3), 5$ (assigned from COSY data)), $6.32(1 \mathrm{H}, \mathrm{t}, \mathrm{Tp}(4)), 6.03(1 \mathrm{H}, \mathrm{t}(J=3.3), 4)$, $1.49\left(9 \mathrm{H}, \mathrm{d}(J=7.8), \mathrm{PMe}_{3}\right) .{ }^{13} \mathrm{C} \mathrm{NMR}\left(\right.$ acetone- $\left.d_{6}, \delta\right): 156.6\left(\mathrm{~s}, 2\left(-80^{\circ} \mathrm{C}\right), 149.5(\mathrm{~s}, 6(-\right.$ $\left.80^{\circ} \mathrm{C}\right)$ ), 144.4, 144.2 (Tp 3 and 5 positions), $142.1\left(\mathrm{~s}, 4\left(-80^{\circ} \mathrm{C}\right)\right), 141.9$ (s, Tp $\left.(3,5)\right)$, $119.5\left(\mathrm{~d}(\mathrm{~J}=5.1), 5\left(-80^{\circ} \mathrm{C}\right)\right), 137.2,137.1,136.9$ (Tp 3 and 5 positions $), 108.1,107.6$, 107.6 (Tp 4 positions), 16.0 ( $\left.\mathrm{d}(\mathrm{J}=27.8), \mathrm{PMe}_{3}\right),{ }^{31} \mathrm{P}$ NMR (acetone- $\left.d_{6}, \delta\right):-8.19$ (satellite $\left.\mathrm{d}(J=412.2), \mathrm{PMe}_{3}\right)$. Estimated to be greater then $90 \%$ pure by NMR and CV data.

\section{TpW(NO)(PMe 3 )(3,4- $\eta^{2}$-Dimethyl-pyrimidin-2-yl-amine) (3)}

Compound 1 (514 mg, $0.88 \mathrm{mmol})$ was added to a solution of 2-(N,N-

dimethyl)aminopyrimidine $(2.02 \mathrm{~g}, 16.4 \mathrm{mmol})$ in $13 \mathrm{~mL}$ of pentane. The suspension was stirred vigorously for 5 days. $50 \mathrm{~mL}$ of additional pentane was added to the reaction mixture and the precipitate was collected on medium porosity glass filter and dried in vacuo to give 3 in a 4.6:1 (A:B) ratio (488 mg, $0.78 \mathrm{mmol}, 88.1 \%$ yield) as a light yellow powder. IR (HATR glaze): $v_{\text {NO }}=1570 \mathrm{~cm}^{-1} . \mathrm{CV}$ (DMA, TBAH, $100 \mathrm{mV} / \mathrm{s}$, vs. NHE): $\mathrm{E}_{\mathrm{p}, \mathrm{a}}$ $=-0.34 \mathrm{~V} .{ }^{1} \mathrm{H}$ NMR (acetone- $\left.d_{6}, \delta\right): 8.01(1 \mathrm{H}, \mathrm{d}, \mathrm{Tp}), 7.97(1 \mathrm{H}, \mathrm{d}, \mathrm{Tp}), 7.93(1 \mathrm{H}, \mathrm{d}, \mathrm{Tp})$, $7.85(1 \mathrm{H}, \mathrm{d}, \mathrm{Tp}), 7.82(1 \mathrm{H}, \mathrm{d}, \mathrm{Tp}(\mathrm{B})), 7.58(1 \mathrm{H}, \mathrm{d}, \mathrm{Tp}), 7.52(1 \mathrm{H}, \mathrm{d}, \mathrm{Tp}(\mathrm{B})), 7.29(1 \mathrm{H}, \mathrm{d}$, Tp), $6.38(1 \mathrm{H}, \mathrm{t}, \mathrm{Tp}(\mathrm{B})), 6.36(1 \mathrm{H}, \mathrm{t}, \mathrm{Tp}), 6.28(1 \mathrm{H}, \mathrm{t}, \mathrm{Tp}), 6.27(1 \mathrm{H}, \mathrm{t}, \mathrm{Tp}), 6.18(1 \mathrm{H}, \mathrm{d}$ $(J=6.9), 6 \mathrm{~A}), 5.53(1 \mathrm{H}, \mathrm{dd}(J=1.5,6.9), 5 \mathrm{~A}), 4.81(1 \mathrm{H}, \mathrm{dd}(J=1.5,18.6), 4 \mathrm{~A}), 2.97$ (3H, s, N-Me (B)), 2.79 (3H, s, N-Me (A)), 1.75 (3H, s, N-Me (A)), 1.37 (9H, d (J=8.7), $\left.\mathrm{PMe}_{3}\right) .{ }^{13} \mathrm{C}$ NMR (acetone- $d_{6}, \delta$ ): $167.7 \pm 1.4$ (s, 2A (assigned from HMBC data)), 145.0, 143.2, 141.7, 137.7, 136.9, 136.7 (Tp 3 and 5 positions), 134.6 (s, 6A), 107.7 (s, 5A), 107.3, 106.7, 106.2 (Tp 4 positions), $71.3(\mathrm{~d}(\mathrm{~J}=12.6), 4 \mathrm{~A}), 35.9(\mathrm{~s}, \mathrm{~N}-\mathrm{Me}), 35.1(\mathrm{~s}, \mathrm{~N}-$ $\mathrm{Me}), 13.4\left(\mathrm{~d}(J=29.5), \mathrm{PMe}_{3}\right) .{ }^{31} \mathrm{P} \mathrm{NMR}$ (acetone- $\left.d_{6}, \delta\right):-10.63$ (satellite $\mathrm{d}(J=312)$, $\mathrm{PMe}_{3}$ ). Estimated to be greater then $90 \%$ pure by NMR and CV data.

\section{TpW(NO) $\left(\mathrm{PMe}_{3}\right)\left(\eta^{2}(2,3)-2,6-D i a z a-b i c y c l o[2.2 .2]\right.$ octa-2,5-diene-8-carboxylic acid methyl ester) (4)}

A homogenous solution of methyl acrylate $(2.007 \mathrm{~g}, 23.31 \mathrm{mmol})$ in $8.2 \mathrm{~mL}$ benzene was added to complex 2 (451 mg, $0.77 \mathrm{mmol})$. The resulting solution was stirred for $120 \mathrm{hrs}$. The reaction solution was then evaporated to a quarter of the original volume under reduced pressure. The concentrated solution was added to $100 \mathrm{~mL}$ of stirring ether. The polymeric precipitate was removed via filtration and the solution was evaporated to an oil under reduced pressure. The oil was redissolved in $3 \mathrm{~mL}$ of $\mathrm{CH}_{2} \mathrm{Cl}_{2}$ and solution was added to $80 \mathrm{~mL}$ of stirring pentane. The precipitate was collected on medium porosity glass filter and dried in vacuo to give crude 4 (335 mg, $0.50 \mathrm{mmol}, 65 \%$ yield) as a light blue powder. Stirring the product overnight in minimal acetone removes trace polymeric impurities. IR (HATR glaze): $v_{\mathrm{NO}}=1552 \mathrm{~cm}^{-1}, v_{\mathrm{CN}}=1641 \mathrm{~cm}^{-1}, v_{\mathrm{CO}}=1729 \mathrm{~cm}^{-1}$. CV (DMA, TBAH, $100 \mathrm{mV} / \mathrm{s}$, vs. NHE): $\mathrm{E}_{\mathrm{p}, \mathrm{a}}=-0.31 \mathrm{~V} .{ }^{1} \mathrm{H}$ NMR (DMF- $\left.d_{7}, \delta\right): 8.21(1 \mathrm{H}, \mathrm{d}$, Tp $(3,5)), 8.17(1 \mathrm{H}, \mathrm{dd}(J=1.4,4.2), 5), 8.10(1 \mathrm{H}, \mathrm{d}, \mathrm{Tp}(3,5)), 8.09(1 \mathrm{H}, \mathrm{d}, \mathrm{Tp}(3,5))$, $7.97(1 \mathrm{H}, \mathrm{d}, \mathrm{Tp}(3,5)), 7.97(1 \mathrm{H}, \mathrm{d}, \mathrm{Tp}(3,5)), 7.84(1 \mathrm{H}, \mathrm{d}, \mathrm{Tp}(3,5)), 6.46(1 \mathrm{H}, \mathrm{t}, \mathrm{Tp}(4))$, $6.31(1 \mathrm{H}, \mathrm{t}, \mathrm{Tp}(4)), 6.27(1 \mathrm{H}, \mathrm{t}, \mathrm{Tp}(4)), 5.64(1 \mathrm{H}, \mathrm{t}(J=2.4), 1), 3.75(1 \mathrm{H}, \operatorname{tdd}(J=1.4$, 2.4, 4.2), 4), 3.55 (3H, s, OMe), 2.65 (1H, ddd $(J=2.4,5.1,10.3), 8), 2.66(1 \mathrm{H}, \operatorname{td}(J=$ 1.4, 3.2), 3), 2.49 (1H, ddd $(J=2.4,5.1,12.5), 7), 2.00\left(1 \mathrm{H}, \mathrm{ddd}(J=2.4,10.3,12.5), 7^{\prime}\right)$, $1.38\left(9 \mathrm{H}, \mathrm{d}(J=8.9), \mathrm{PMe}_{3}\right) .{ }^{13} \mathrm{C} \mathrm{NMR}\left(\mathrm{DMF}_{-} d_{7}, \delta\right): 173.9(\mathrm{~s}, 9), 168.0(\mathrm{~s}, 5), 144.8$, 144.5, 144.1, 137.2, 136.7, 135.5 (Tp 3 and 5 positions), 106.7, 106.4, 105.7 (Tp 4 
positions), $72.7(\mathrm{~s}, 1), 64.9$ (s, 3), $51.4(\mathrm{~s}, \mathrm{OMe}), 42.5$ (s, 8 or 4), 42.3 (s, 8 or 4), 31.7 (s, 7), 13.3 (d $\left.(J=28.7), \mathrm{PMe}_{3}\right) .{ }^{31} \mathrm{P}$ NMR $\left(\mathrm{DMF}-d_{7}, \delta\right):-11.06$ (satellite d $\left.(J=291), \mathrm{PMe}_{3}\right)$. Calc. for $\mathrm{C}_{20} \mathrm{H}_{29} \mathrm{BN}_{9} \mathrm{O}_{3} \mathrm{PW}$ : C, 35.9; H, 4.37; N, 18.84. Found: C, 35.74; H, 4.35; N, 18.72 .

\section{TpW(NO)(PMe3)(2,3- $\eta^{2}$-2,6-Diaza-bicyclo[2.2.2] octa-2,5-diene-8-carbonitrile) (5)}

Neat acrylonitrile (3.086 g, $5.82 \mathrm{mmol})$ was added to complex 2 (229 mg, $0.39 \mathrm{mmol})$ and stirred for $12 \mathrm{hrs}$. The reaction solution was added to $100 \mathrm{~mL}$ of stirring ether. The polymeric precipitate was removed via filtration and the solution was evaporated to an oil under reduced pressure. The oil was redissolved in $5 \mathrm{~mL}$ of benzene and added to $100 \mathrm{~mL}$ of stirring pentane. The precipitate was collected on a medium porosity glass filter and dried in vacuo to give 5 (A:B:C:D=4:6:3:1) (139 mg, $0.22 \mathrm{mmol}, 56 \%$ yield) as a light green powder. IR (HATR glaze): $v_{\mathrm{NO}}=1553 \mathrm{~cm}^{-1}, v_{\mathrm{NC}}=2241 \mathrm{~cm}^{-1}$. CV (DMA, TBAH, $100 \mathrm{mV} / \mathrm{s}$, vs. NHE): $\mathrm{E}_{\mathrm{p}, \mathrm{a}}=+0.46 \mathrm{~V} .{ }^{1} \mathrm{H}$ NMR (acetone- $\left.d_{6}, \delta\right): 8.18(1 \mathrm{H}, \mathrm{d}, \mathrm{Tp}(3,5)), 8.12$ $\pm 0.01(1 \mathrm{H}$, dd (buried), 5(B) (assigned by COSY data)), $8.11(1 \mathrm{H}, \mathrm{dd}(J=1.5,4.2)$, $5(\mathrm{~A})), 8.10 \pm 0.02(1 \mathrm{H}, \mathrm{d}, \mathrm{Tp}(3,5)$ (assigned by COSY data)), $8.07(1 \mathrm{H}, \mathrm{d}, \mathrm{Tp}(3,5))$, $8.05 \pm 0.02(1 \mathrm{H}$, dd (buried), 5(D) (assigned by COSY data)), $8.04(1 \mathrm{H}, \mathrm{d}, \mathrm{Tp}(3,5)), 8.01$ $(1 \mathrm{H}, \mathrm{d}, \mathrm{Tp}(3,5)), 7.97(2 \mathrm{H}, \mathrm{d}, \mathrm{Tp}(3,5)), 7.95(1 \mathrm{H}, \mathrm{d}, \mathrm{Tp}(3,5)), 7.93(1 \mathrm{H}, \mathrm{d}, \mathrm{Tp}(3,5))$, $7.88(1 \mathrm{H}, \mathrm{d}, \mathrm{Tp}(3,5)), 7.86(2 \mathrm{H}, \mathrm{d}, \mathrm{Tp}(3,5)), 7.77(1 \mathrm{H}, \mathrm{d}, \mathrm{Tp}(3,5)), 7.72(1 \mathrm{H}, \mathrm{d}, \mathrm{Tp}$ $(3,5)), 7.39(2 \mathrm{H}, \mathrm{d}, \mathrm{Tp}(3,5)), 6.40(1 \mathrm{H}, \mathrm{t}, \mathrm{Tp}(4)), 6.38(1 \mathrm{H}, \mathrm{t}, \mathrm{Tp}(4)), 6.31(1 \mathrm{H}, \mathrm{t}, \mathrm{Tp}$ (4)), $6.28(1 \mathrm{H}, \mathrm{t}, \mathrm{Tp}(4)), 6.25(1 \mathrm{H}, \mathrm{t}, \mathrm{Tp}(4)), 6.23(1 \mathrm{H}, \mathrm{t}, \mathrm{Tp}(4)), 6.22(1 \mathrm{H}, \mathrm{t}, \mathrm{Tp}(4))$, $6.20(1 \mathrm{H}, \mathrm{t}, \mathrm{Tp}(4)), 5.68(1 \mathrm{H}, \mathrm{t}(J=2.4), 1(\mathrm{C})), 5.65(1 \mathrm{H}, \mathrm{t}(J=2.4), 1(\mathrm{~A})), 5.53(1 \mathrm{H}, \mathrm{t}(J$ $=2.3), 1(\mathrm{~B})), 5.49(1 \mathrm{H}, \mathrm{t}(J=2.3), 1(\mathrm{D})), 4.25(1 \mathrm{H}, \mathrm{td}(J=1.2,15.4), 3(\mathrm{D})), 3.90(1 \mathrm{H}, \mathrm{td}$ $(J=1.3,15.1), 3(\mathrm{~B})), 3.79(1 \mathrm{H}, \mathrm{m}, 4(\mathrm{~B})), 3.74(1 \mathrm{H}, \mathrm{m}, 4(\mathrm{C})), 3.64(1 \mathrm{H}, \mathrm{m}, 4(\mathrm{D}$ and A)), $3.02(1 \mathrm{H}$, ddd $(J=2.2,4.0,10.0), 8(\mathrm{~B})), 3.00(1 \mathrm{H}, \mathrm{td}(J=1.5,3.4), 3(\mathrm{~A})), 2.87 \pm 0.03$ (1H, m (buried), 8(C) (assigned by COSY data)), $2.85(1 \mathrm{H}$, ddd $(J=2.4,6.0,9.6), 8(\mathrm{~A}))$, $2.76(1 \mathrm{H}, \mathrm{m}, 8(\mathrm{D})), 2.62(1 \mathrm{H}, \mathrm{td}(J=1.5,3.3), 3(\mathrm{C})), 2.55(1 \mathrm{H}, \mathrm{ddd}(J=2.3,10.0,12.3)$, 7(C)), 2.30 (1H, ddd $(J=2.3,10.0,12.5), 7(\mathrm{~B})), 2.27(1 \mathrm{H}, \mathrm{m}$ (buried), 7(A)), $2.25(1 \mathrm{H}, \mathrm{m}$ (buried), 7'(A)), 2.19 (1H, m (buried), 7(D)), 1.98 (1H, ddd $\left.(J=2.2,5.1,12.6), 7^{\prime}(\mathrm{D})\right)$, $1.96\left(1 \mathrm{H}, \mathrm{m}\right.$ (buried), $\left.7^{\prime}(\mathrm{C})\right), 1.88\left(1 \mathrm{H}, \mathrm{ddd}(J=2.3,4.0,12.5), 7^{\prime}(\mathrm{B})\right), 1.41(9 \mathrm{H}, \mathrm{d}(J=$ 8.8), PMe3(D)), 1.40 (9H, d $(J=8.8)$, PMe3(B)), 1.37 (9H, d $(J=8.9)$, PMe3(A)), 1.36 $(9 \mathrm{H}, \mathrm{d}(J=8.9), \mathrm{PMe} 3(\mathrm{C})) .{ }^{13} \mathrm{C}$ NMR (acetone- $\left.d_{6}, \delta\right): 168.7$ (s, 5(D)), 168.1 (s, 5(A or B)), 167.8 (s, 5(A or B)), 167.2 (s, 5(C)), 145.4, 145.1, 145.1, 144.9, 144.9, 144.2, 137.3, 137.5, 137.2, 136.7, 136.0 (Tp 3 and 5 positions), 123.7 (s, 9(A or B)), 122.8 (s, 9(A or B)), 107.2, 107.0, 106.8, 106.7, 106.6, 106.5, 106.3, 106.2 (Tp 4 positions), 72.3 (s, 1(A)), 70.4 (s, 1(B)), 70.3 (s, 1(D)), 68.7 (s, 3(C)), 67.3 (d (J= 16.5), 3(B)), 64.8 (s, 3(A)), 63.8 (d (J= 16.7), 3(D)), 43.9 (s, 4(B)), 43.3 (s, 4(C)), 42.4 (s, 4(D)), 42.0 (s, 4(A)), 35.2 (s, 7(C)), 34.7 (s, 7(A)), 34.4 (s, 7(B)), 33.8 (s, 7(D)), 28.9 (s, 8(A and B)), 28.2 (s, 8(D)), 13.9 (d $(J=28.5), \operatorname{PMe} 3(\mathrm{~A})), 13.5$ (d $\left.(J=30.6), \mathrm{PMe}_{3}(\mathrm{~B})\right), 13.3$ (d $(J=$ 29.9), $\left.\mathrm{PMe}_{3}(\mathrm{D})\right) .{ }^{31} \mathrm{P}$ NMR (acetone- $\left.d_{6}, \delta\right):-11.06$ (satellite d $\left.(J=279.6), \mathrm{PMe}_{3}\right),-11.43$ (satellite d $\left.(J=279.3), \mathrm{PMe}_{3}\right),-12.46$ (satellite d $\left.(J=289), \mathrm{PMe}_{3}\right)$. HRMS: Calc: 653.17917 (10), 654.18168 (10), 655.18150 (12), 656.18567 (5), 657.18477 (10); Found: 653.18126 (10), 654.18531 (10), 655.18155 (12), 656.18626 (5), 657.18391(10).

Estimated to be greater then $90 \%$ pure by $\operatorname{NMR}\left({ }^{1} \mathrm{H},{ }^{13} \mathrm{C},{ }^{31} \mathrm{P}\right)$, IR, and $\mathrm{CV}$ data. 


\section{${ }^{1}$ H NMR for compound 2:}

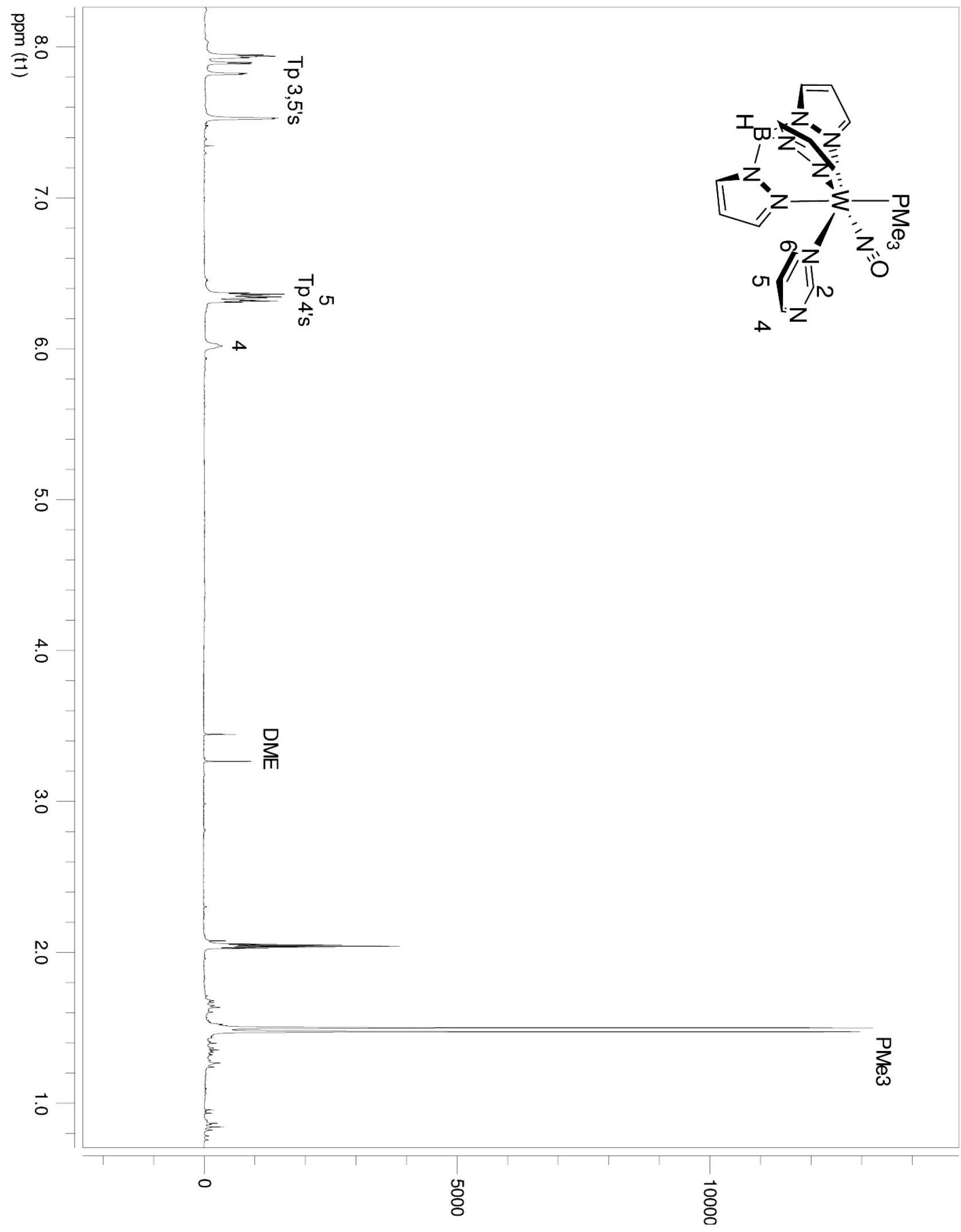




\section{${ }^{13} \mathrm{C}$ NMR for compound 2:}

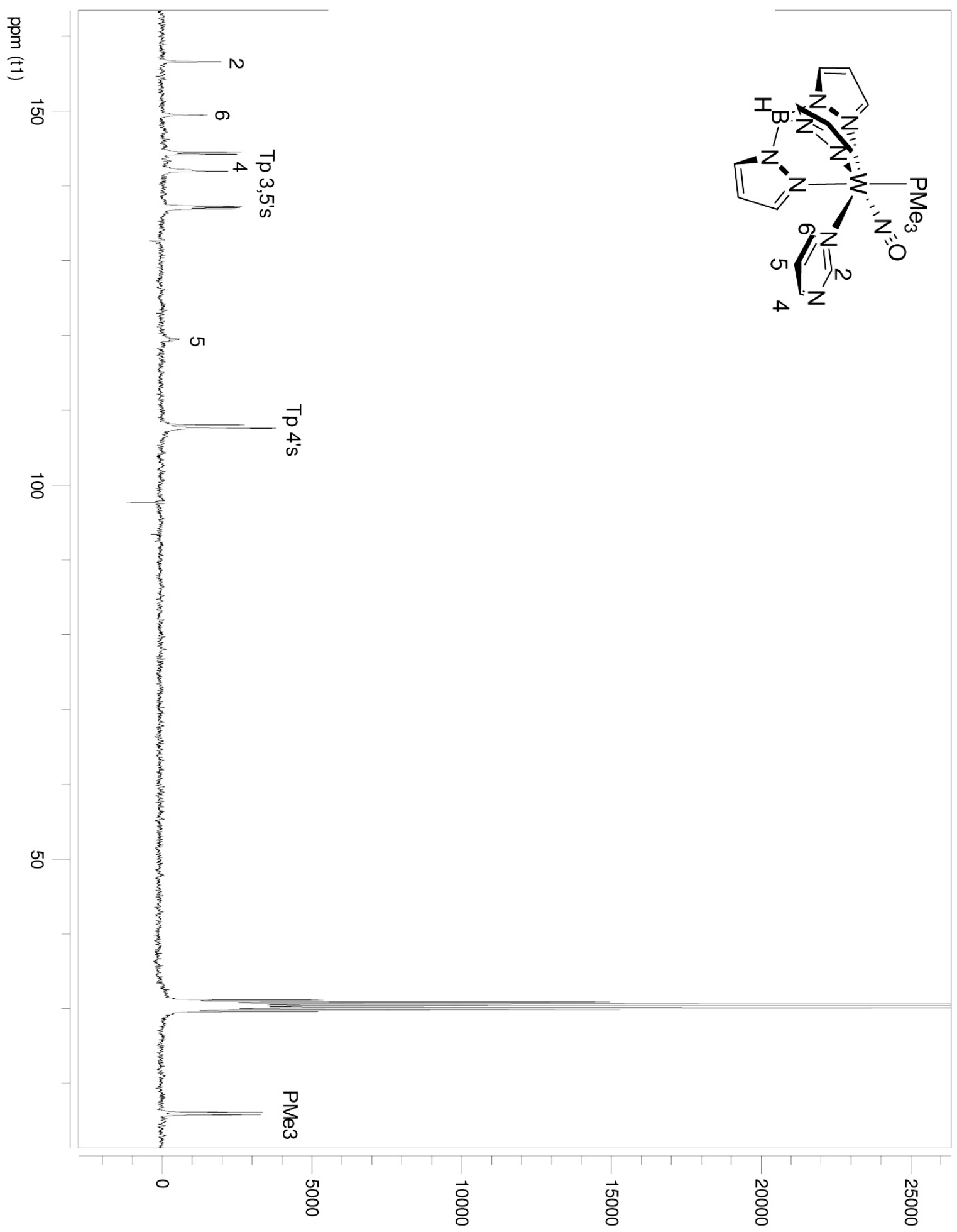




\section{${ }^{1} \mathrm{H}$ NMR for compound 3:}

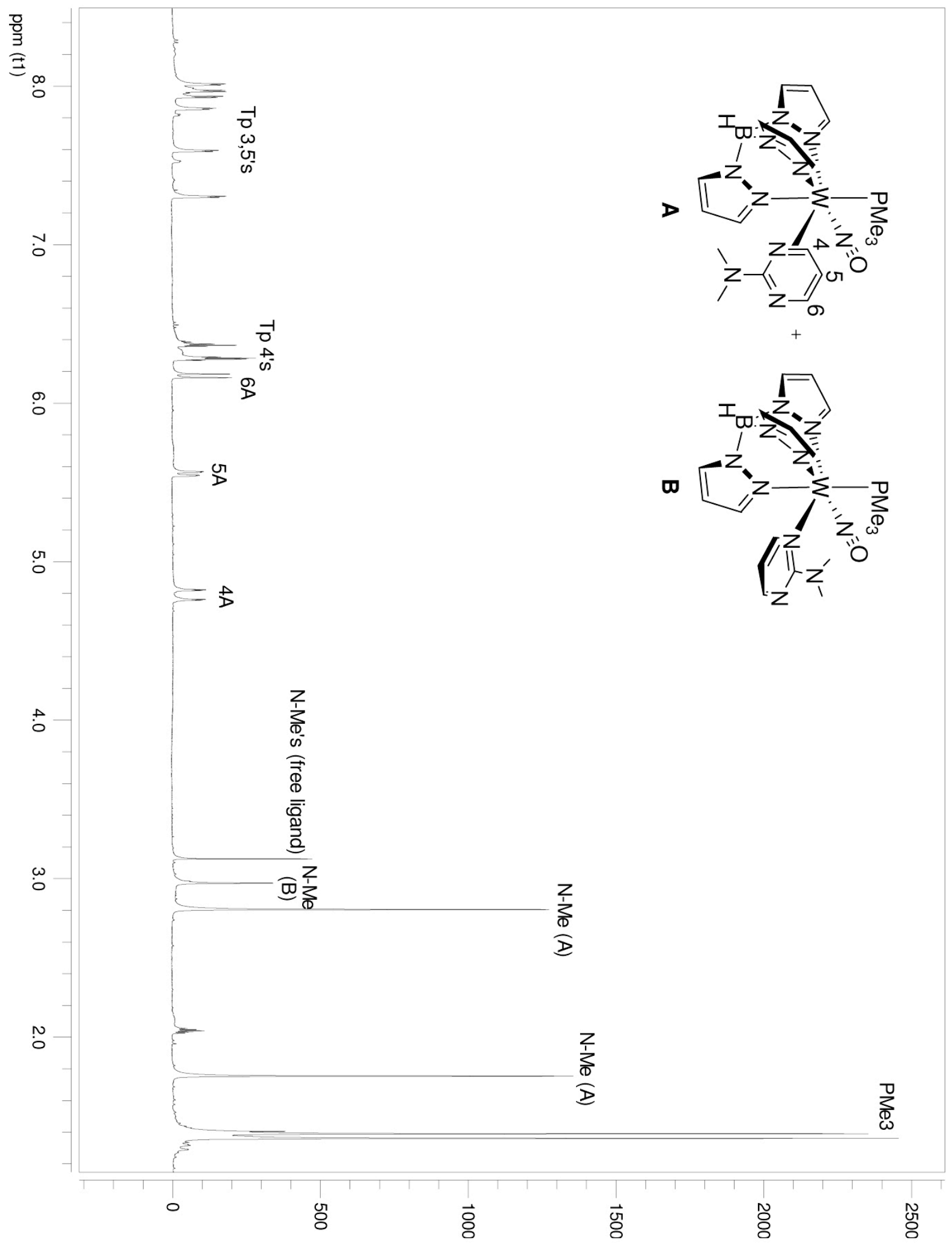




\section{${ }^{13} \mathrm{C}$ NMR for compound 3:}

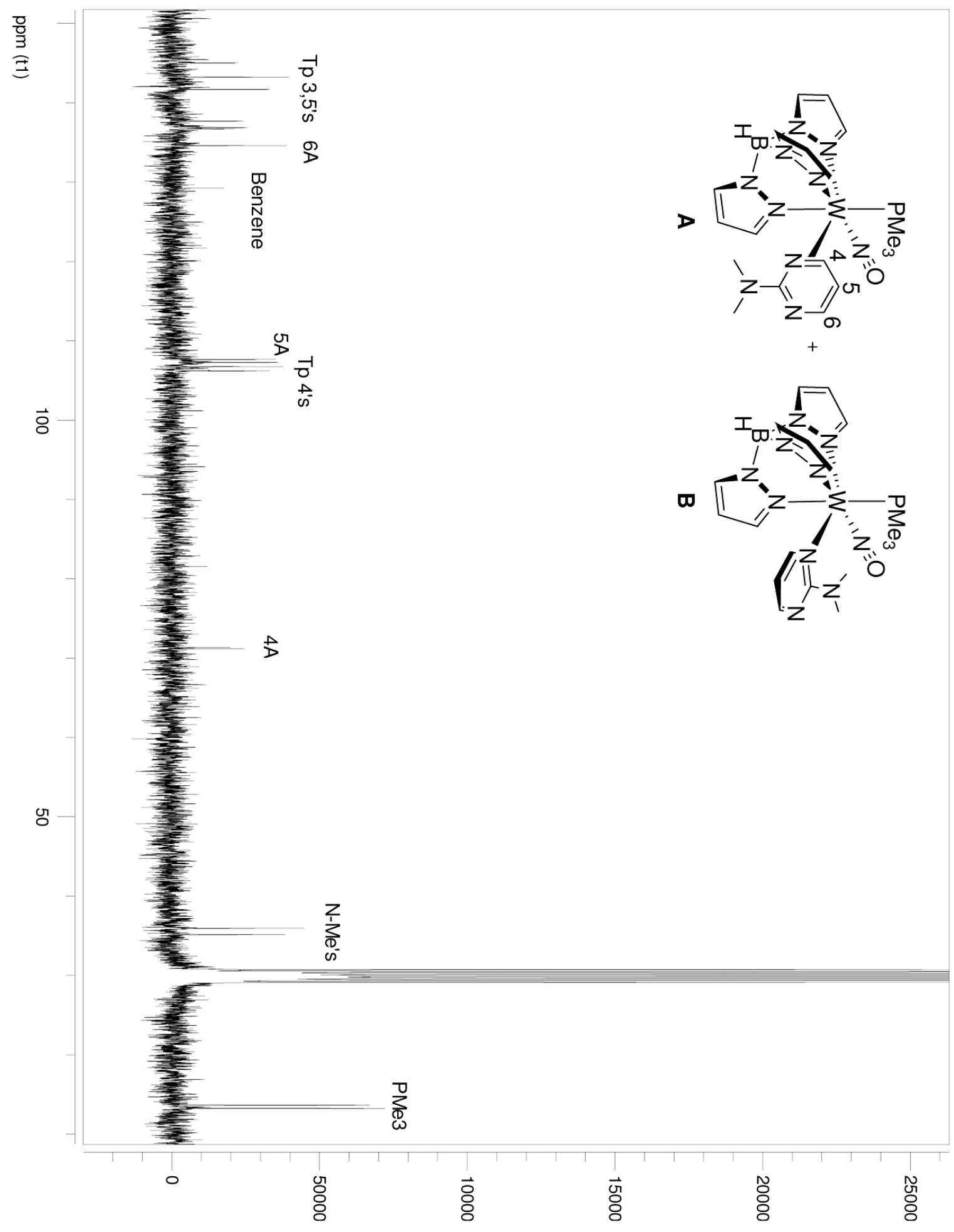




\section{${ }^{1} \mathrm{H}$ NMR for compound 4:}

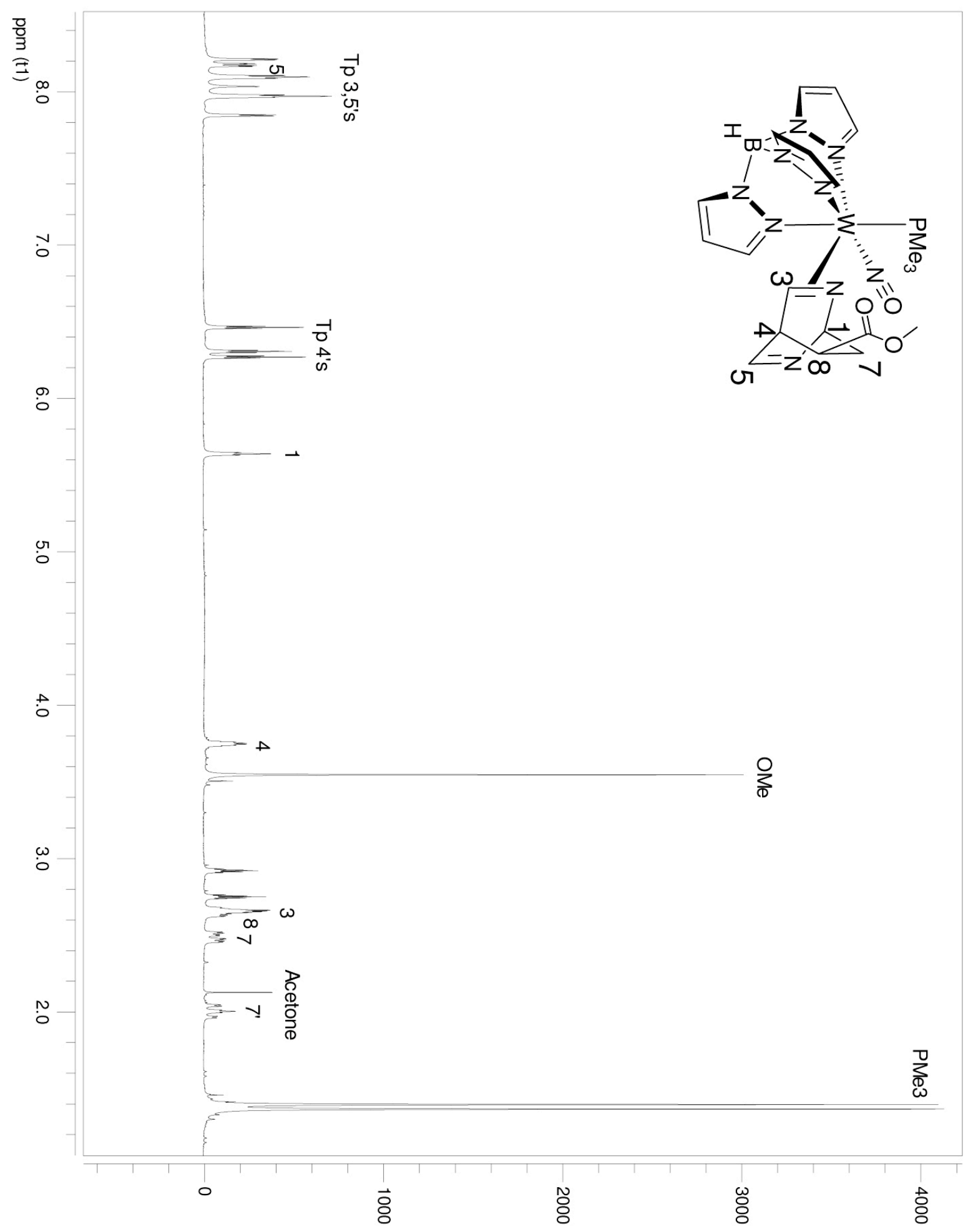




\section{${ }^{13} \mathrm{C}$ NMR for compound 4:}

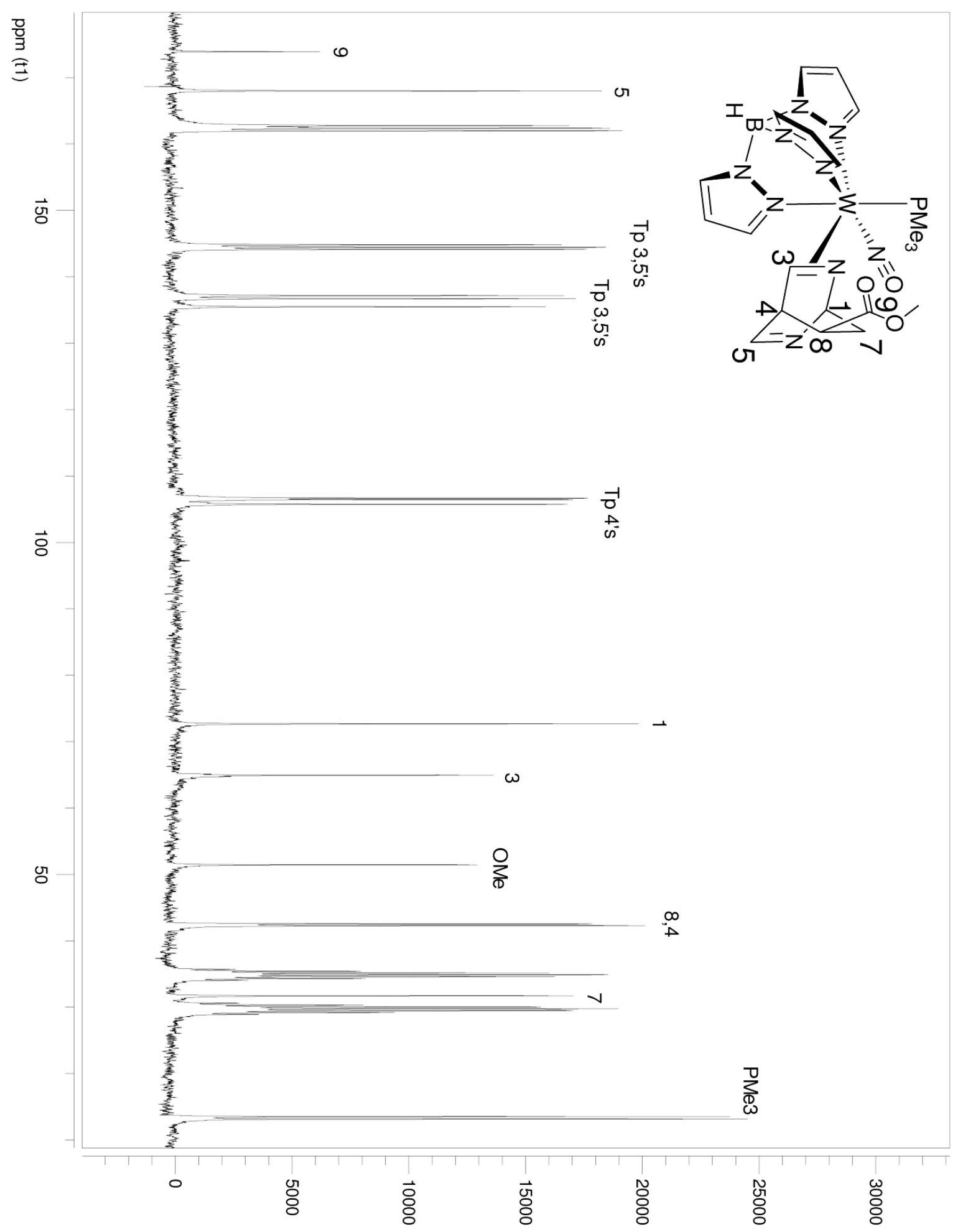




\section{${ }^{1}$ H NMR for compound 5:}

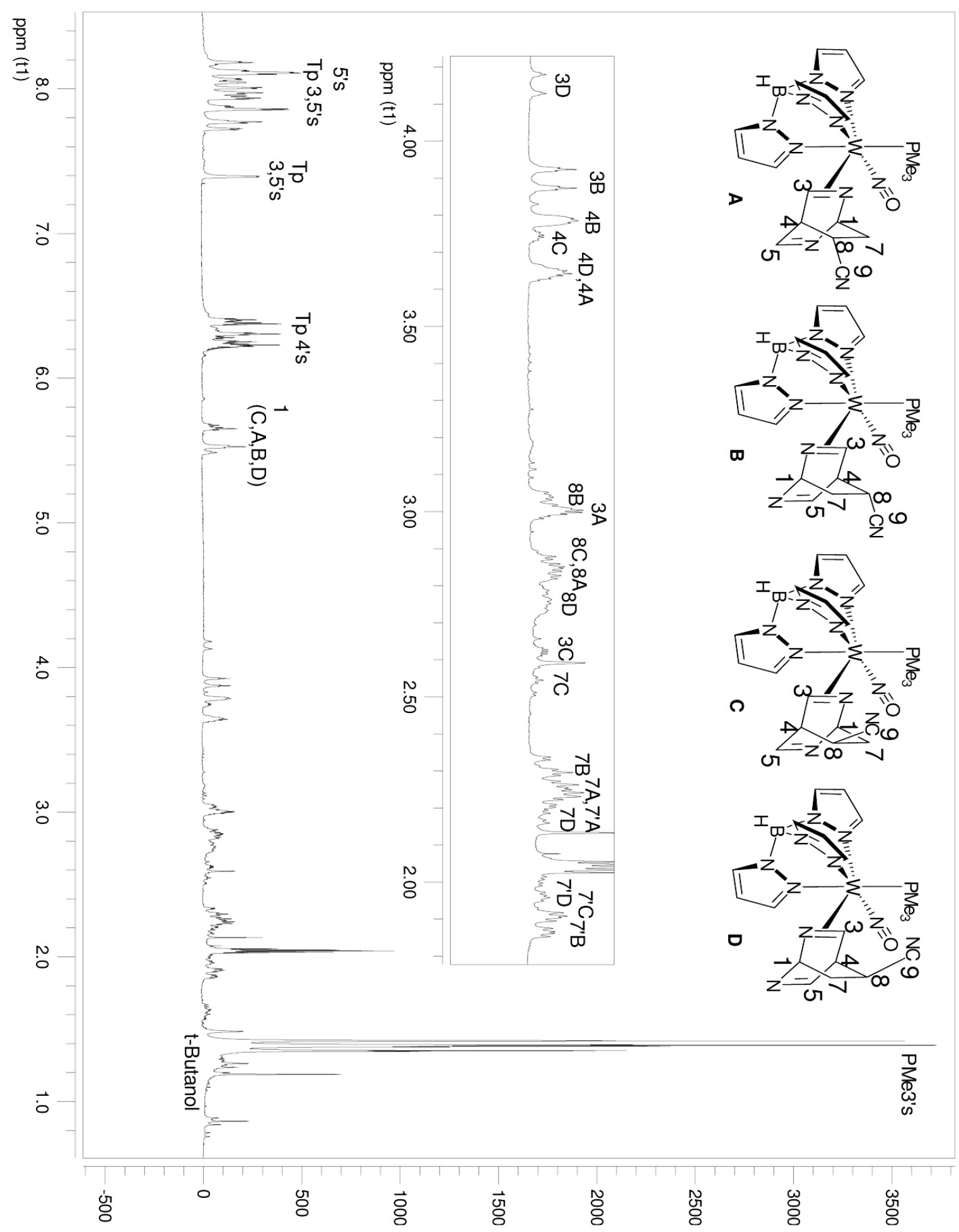




\section{${ }^{13}$ C NMR for compound 5:}

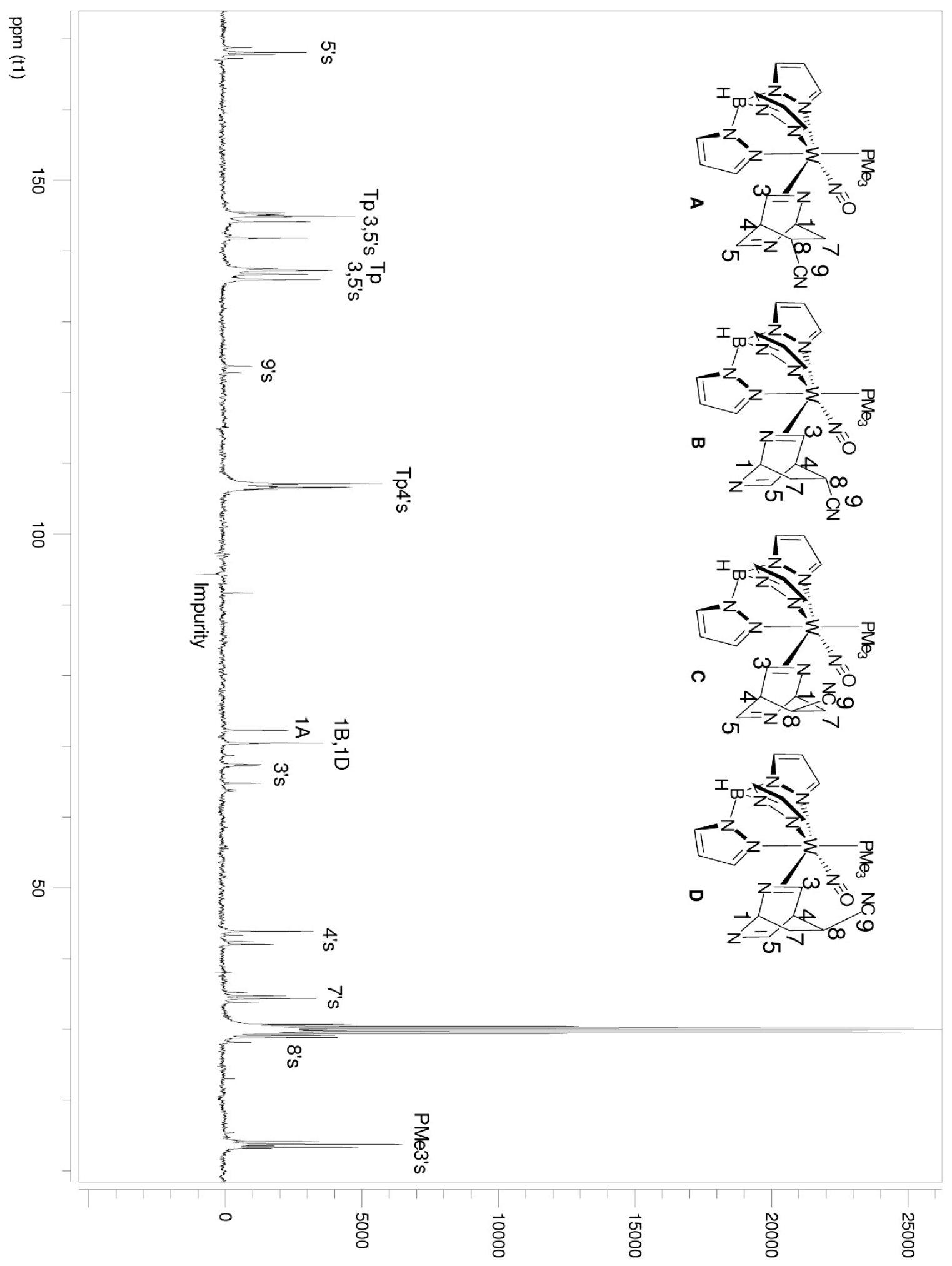

